\title{
Metagenomic analysis of acquired antibiotic resistance determinants in the gut microbiota of wild boars (Sus scrofa) - preliminary results
}

\author{
Balázs Libisch ${ }^{1}$, Tibor Keresztény ${ }^{1}$, Zoltán Kerényi ${ }^{2}$, Róbert Kocsis ${ }^{2}$, \\ Rita Sipos ${ }^{3}$, Péter P. Papp ${ }^{1}$, Ferenc Olasz ${ }^{1}$ \\ ${ }^{1}$ Laboratory of Microbiology, Agricultural Biotechnology Institute, \\ National Agricultural Research and Innovation Centre (NARIC), 2100 Gödöllö, Hungary \\ ${ }^{2}$ Hungarian Dairy Research Institute Ltd., 9200 Mosonmagyaróvár, Hungary \\ ${ }^{3}$ BIOMI Ltd., 2100 Gödöllő, Hungary \\ balazs.libisch@freemail.hu
}

Received: June 3, 2019

Accepted: February 13, 2020

\begin{abstract}
Introduction: Land application of manure that contains antibiotics and resistant bacteria may facilitate the establishment of an environmental reservoir of antibiotic-resistant microbes, promoting their dissemination into agricultural and natural habitats. The main objective of this study was to search for acquired antibiotic resistance determinants in the gut microbiota of wild boar populations living in natural habitats. Material and Methods: Gastrointestinal samples of free-living wild boars were collected in the Zemplén Mountains in Hungary and were characterised by culture-based, metagenomic, and molecular microbiological methods. Bioinformatic analysis of the faecal microbiome of a hunted wild boar from Japan was used for comparative studies. Also, shotgun metagenomic sequencing data of two untreated sewage wastewater samples from North Pest (Hungary) from 2016 were analysed by bioinformatic methods. Minimum spanning tree diagrams for seven-gene MLST profiles of 104 E. coli strains isolated in Europe from wild boars and domestic pigs were generated in Enterobase. Results: In the ileum of a diarrhoeic boar, a dominant E. coli O112ab:H2 strain with intermediate resistance to gentamicin, tobramycin, and amikacin was identified, displaying sequence type ST388 and harbouring the EAST1 toxin ast $A$ gene. Metagenomic analyses of the colon and rectum digesta revealed the presence of the tet $Q$, tet $W$, tet $O$, and mefA antibiotic resistance genes that were also detected in the gut microbiome of four other wild boars from the mountains. Furthermore, the tet $Q$ and $c f x A$ genes were identified in the faecal microbiome of a hunted wild boar from Japan. Conclusion: The gastrointestinal microbiota of the free-living wild boars examined in this study carried acquired antibiotic resistance determinants that are highly prevalent among domestic livestock populations.
\end{abstract}

Keywords: wild boar, intestinal microbiota, antibiotic resistance, tetracycline, natural habitat.

\section{Introduction}

The wild boar population in Hungary and elsewhere in Europe has shown substantial growth in the past decades. Hungarian spring population data indicated 58,900 and 101,682 wild boars in 1997 and 2016, respectively, according to the National Game Management Database (3, 7). Increased density and spatial aggregation of wild boars in fenced and fed hunting estates increases the risk of animals coming into contact with multiple disease agents, where these situations are examples of how overabundance might affect animal health.

Antibiotics are widely used in human medicine for disease prevention and in veterinary medicine for treatment of animals; in the latter field, they have also been applied for promoting growth in animal farming. The breadth of their use has led to the emergence of antibiotic-resistant strains in the gut microbiota. Land application of sewage sludge or animal manure that contains antibiotics and resistant bacteria facilitates the establishment of an environmental reservoir and the selection of antibiotic-resistant microbes (12), promoting 
their dissemination into agricultural and natural habitats and wild animal populations. It has been suggested that European wild boars are frequent carriers of antimicrobial-resistant bacteria, such as E. coli and salmonellae, and are likely to be involved in the transmission of antimicrobial resistance throughout the environment (30). Wild boars feeding on agricultural lands bordering forested areas might at least in part contribute to this phenomenon, and furthermore, wild boars may accumulate antibiotic-resistant bacteria derived from their diverse diet (27).

Earlier studies mainly assessed the antibiotic susceptibility of cultured isolates of particular bacterial species in the gut microbiota of wild boars (such as E. coli, Salmonella spp., Enterococcus spp., and Lactobacillus spp.), and identified various acquired antibiotic resistance determinants, including those for tetracyclines, aminoglycosides, ß-lactams, sulphonamides, and chloramphenicol $(3,14,16,27)$. In this study, the gastrointestinal microbiota of free-living wild boars from Hungary and Japan were analysed by a complex approach with the objective of searching for and identifying acquired antibiotic resistance determinants in free-living wild boar populations in natural habitats. The term "resistome" was defined as the full collection of resistance genes in microorganisms and microbial populations (25). Instead of examining the antibiotic resistance of certain cultured bacterial isolates, the application of shotgun metagenomic methods may provide a more comprehensive approach to characterising the acquired resistome in the gut microbiota of wild animal populations. During this work targeting freeliving wild boars, a gentamicin non-susceptible $E$. coli strain was also characterised from the ileal digesta of a diarrhoeic animal.

\section{Material and Methods}

Sample collection and processing. Intestine segments of the ileum, colon, and rectum with digesta content were collected from an adult female diarrhoeic wild boar (Sus scrofa) shot during the regular hunting season in November 2016 in the Zemplén Mountains in North-East Hungary, about four kilometres from the Slovakian border. Gastrointestinal samples were subsequently collected from four additional hunted freeliving wild boars in the Zemplén Mountains in January 2017 , including two adult female, one juvenile male, and one juvenile female. Gastrointestinal samples were transported to the laboratory under refrigerated and anaerobic conditions using anaerobic jars supplemented with AnaeroGen 2.5L sachets (Oxoid, Basingstoke, UK). The samples were processed within $24 \mathrm{~h}$. The rectal content of a hunted free-living wild boar was collected in Japan in 2013 and was subjected to shotgun metagenomic sequencing by Ushida et al. (24) (NCBI BioSample SAMD00022871). The sequencing data from Japan was also examined in the study so that both the European and Asian clades of the Eurasian wild boar
(Sus scrofa) were included in the gut metagenomic analyses. Also, two untreated sewage wastewater samples from North Pest (Hungary) were collected in 2016 and subjected to shotgun metagenomic sequencing by a global consortium represented by the Centre for Genomic Epidemiology, National Food Institute, Technical University of Denmark (NCBI SRA codes ERR2607463 and ERR1713361).

DNA isolation from digesta samples and metagenomic sequencing. Total DNA was extracted from the ileum, colon, and rectum content of the diarrhoeic wild boar from the Zemplén Mountains by the QIAamp DNA Stool Mini Kit (Qiagen, Hilden, Germany). DNA samples were shipped to Xenovea Ltd. (Szeged, Hungary), a commercial service provider for amplicon (V3-V4 variable regions of the 16S rRNA gene) and shotgun metagenomic sequencing on Illumina (Santa Clara, CA, USA) MiSeq and NextSeq500 platforms, respectively. The numbers of $16 \mathrm{~S} \mathrm{V3-V4}$ merged reads passing the quality control steps and assigned to the bacteria kingdom were 17,276, 18,165, and 16,554 for the ileum, colon, and rectum, respectively. Taxonomic analysis was performed by pipeline v.3.0 of the EBI Metagenomics platform (https://www.ebi.ac.uk/metagenomics). Metagenomic pairedend $2 \times 150 \mathrm{bp}$ shotgun reads totalling 38.4 million, 44.4 million, and 39.8 million were analysed for the ileum, colon, and rectum content, respectively. Furthermore, total DNA was also extracted from gastrointestinal samples of four additional wild boars from the Zemplén Mountains in January 2017. Metagenomic contig assembly was performed by metaSPAdes v3.12.0 (https://omictools.com/metaspades-tool), MEGAHIT v1.1.1 (https://omictools.com/ megahit-tool) and Unicycler v0.4.1.1 (https://omictools.com/unicycler-tool). The wild boar gastrointestinal samples collected in this study and their metagenomic sequencing reads were registered in the European Nucleotide Archive (ENA) under sample IDs ERS1753160, ERS1753161 and ERS1753162.

Culturing and identification of $E$. coli isolates. Enterobacterial strains were cultured from the ileal digesta of the diarrhoeic boar on violet red bile glucose (VRBG) agar plates (Biolab, Budapest, Hungary). The obtained isolates were identified at the species level using an E. coli-specific PCR (11) and partial sequencing of the $16 \mathrm{~S}$ rRNA and $r p o B$ genes.

$D e$ novo genome sequencing of $E$. coli isolate FKI-5325. Genomic DNA was purified from isolate FKI-5325 with the Qiagen QIAamp DNA Mini Kit according to the manufacturer's instructions. De novo genome sequencing was provided by Enviroinvest Corp. (Pécs, Hungary) using the Illumina MiSeq platform and $2 \times 250$ bp paired-end reads at 183-fold genome coverage. De novo draft genome assembly was performed by SPAdes v3.0.0 (http://cab.spbu.ru/ software/spades/). The sequencing reads of this Whole Genome Shotgun project were deposited at GenBank under accession numbers SRP142223, BioProject PRJNA451389, and BioSample SAMN08969247. The sequencing reads were uploaded to the EnteroBase 
Escherichia/Shigella database (2) under strain code ESC JA4692AA.

Detection of virulence and antibiotic resistance genes. The ResFinder 3.1, VirulenceFinder 2.0, and MyDbFinder 1.2 pipelines (https://cge.cbs.dtu.dk/ services) and the Comprehensive Antibiotic Resistance Database (https://card.mcmaster.ca) were used for the identification of acquired antibiotic resistance genes and enterobacterial virulence factors in the $E$. coli genomic contigs and the wild boar gut metagenomic contigs. Furthermore, 82.5 million $2 \times 150$ bp paired-end shotgun metagenomic reads of the faecal microbiome of a free-living wild boar sampled in 2013 in Japan (NCBI SRA code DRR025070) (24) were also analysed in our study by the above bioinformatic methods, together with shotgun metagenomic reads of two wastewater metagenomes of the North Pest wastewater treatment plant in Hungary from 2016, with NCBI SRA codes ERR1713361 and ERR2607463.

The following oligonucleotide primers were used for the validation of gut metagenomic contig assembly, and for the detection and Sanger sequencing of antibiotic resistance genes in faecal DNA samples of wild boars: tetQ-F: CTGCAAATCCCGACAATTATATT, tetQ-R: GGAAAAGGACAAAGACGGGT, tetO-F: TGTGGA TACTACAACGCATGA, tetO-R: TACGGGTTTTGC TCCCACAA, tetW-F: CATGTAGACGCTGGAAAGAC, tetW-R: TCGCTGGGAAGGATAACAATTT, mefA-F: ATGGAAAAATACAACAATTGGAAAC, mefA-R: CAAAGACTGACTATAGCCTGC, tetQME-F: GTA ATCGTTATGCGGCAGTAA, tetQME-R: ACAATG TATTCAGAGCGGATAT, tetOME-F: AGTGTTTTG GGGCTATTGGAGT, tetO-R: TACGGGTTTTGC TCCCACAA, tetWME-F: GGTGTATGCTGTATA TGTGTATG, and tetW-R: TCGCTGGGAAGGATA ACAATTT. The PCR thermal profile included 1 cycle at $94^{\circ} \mathrm{C}$ for $3 \mathrm{~min} ; 35$ cycles at $94^{\circ} \mathrm{C}$ for $0.5 \mathrm{~min}$, primer-specific annealing temperatures for $0.5 \mathrm{~min}$, $72^{\circ} \mathrm{C}$ for 1 or $2 \mathrm{~min}$; and 1 cycle at $72^{\circ} \mathrm{C}$ for $7 \mathrm{~min}$.

Random amplification of polymorphic DNA (RAPD). RAPD of E. coli isolates obtained on VRBG agar plates was performed using primer 1254 and the protocol as described previously (19). Reaction products were analysed by electrophoresis on $1.5 \%$ agarose gels stained with ethidium bromide. Banding patterns were evaluated by visual inspection and by GelAnalyzer 2010 (http://www.gelanalyzer.com/). RAPD patterns were considered different when the profiles differed by at least one band, and the Dice coefficient was used to quantify similarities between banding patterns.

In silico serotyping and multi locus sequence typing (MLST). In silico serotyping of E. coli FKI-5325 strain was performed by SerotypeFinder 1.1 (https://cge. cbs.dtu.dk/services/) and by the BioNumerics E. coli functional genotyping tool (Applied Maths, SintMartens-Latem, Belgium). In silico MLST was completed using the seven-locus MLST scheme of Achtman (28) at the EnteroBase database (2). The Achtman seven-locus MLST scheme is based on nucleotide sequencing of internal gene fragments of the adk (adenylate kinase), fum C (fumarate hydratase), gyrB (DNA gyrase), icd (isocitrate/isopropylmalate dehydrogenase), $m d h$ (malate dehydrogenase), purA (adenylosuccinate dehydrogenase), and recA (DNA recombination/repair protein) genes. Minimum spanning tree diagrams of the MLST data were generated by the MSTree V2 algorithm and visualised by GrapeTree in EnteroBase (2, 29). A minimum spanning tree represents a set of edges (connections) that link together the nodes (sequence types obtained by the seven-locus MLST) by the shortest possible distance (29).

Genetic relationship investigation among $E$. coli isolates from wild boars and domestic pigs in Europe. In silico MLST typing was performed in EnteroBase based on seven-gene MLST profiles of $104 \mathrm{E}$. coli strains isolated in Europe from wild boars and domestic pigs $(2,28,29)$. Countries of origin (and number of strains) were as follows: Denmark (48), Hungary (8), Czech Republic (7), Spain (7), Ireland (6), Luxembourg (5), Italy (4), Switzerland (3), United Kingdom (3), Croatia (2), France (2), Germany (2), Portugal (2), Austria (1), Belgium (1), Bulgaria (1), Netherlands (1), and Sweden (1). A minimum spanning tree diagram was generated.

Antibiotic susceptibility testing. Antibiotic susceptibility testing of the E. coli FKI-5325 strain was performed by the agar dilution method using Mueller-Hinton agar (Sigma-Aldrich, St. Louis, MO, USA) for gentamicin, ampicillin, ciprofloxacin, nalidixic acid, tetracycline, and chloramphenicol, and by the disc diffusion method using gentamicin $(10 \mu \mathrm{g})$, tobramycin $(10 \mu \mathrm{g})$, and amikacin $(30 \mu \mathrm{g})$ discs (BioRad, Hercules, CA, USA). Minimal inhibitory concentration (MIC) data and results of the disc diffusion tests were interpreted according to the breakpoints defined by the performance standards for antimicrobial susceptibility testing of the Clinical and Laboratory Standards Institute (CLSI) (6). The sensitivity of a bacterial strain to a given antibiotic is interpreted as "intermediate" when it is inhibited in vitro by a concentration of the concerned drug that is associated with an uncertain therapeutic effect (6). The E. coli ATCC 25922 and P. aeruginosa ATCC 27853 strains were used as reference strains.

\section{Results}

Gastrointestinal sample collection. A free living wild boar was sampled in the Zemplén Mountains in Hungary in November 2016. Physical anomalies characteristic for diarrhoea were observed and recorded during the sampling: various parts of its ileum were red (hyperaemic) with a very thin or missing mucosal layer, and the consistency of the faeces (i.e. the rectum content) was watery and its colour grey. Four further free-living wild boars were subsequently sampled in January 2017 in the same place. The boars showed no symptoms of gastrointestinal or any other disease. 
Aminoglycoside non-susceptible $E$. coli strain in the ileum of the diarrhoeic wild boar. Phylum level taxonomic analyses in three sections of the gastrointestinal tract of the diarrhoeic wild boar from the Zemplén Mountains revealed an over 75\% abundance of the proteobacteria in its ileal microbiota, compared to about $10 \%$ established earlier in the ileum of domestic pigs (Fig. 1) (13). Ninety-nine percent of proteobacteria were assigned to the family Enterobacteriaceae in the ileum of the diarrhoeic wild boar.

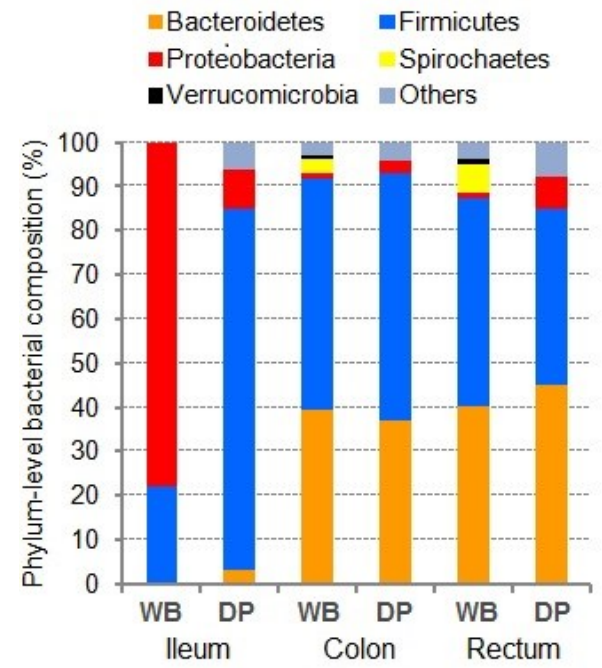

Fig. 1. Phylum-level bacterial composition of the microbiota in three sections of the gastrointestinal tract of the diarrhoeic wild boar (WB) from Hungary analysed in this work in comparison with those established earlier for the domestic pig (DP) by a meta-analysis (13)

A dominant E. coli O112ab:H2 strain was identified in the ileal microbiota of the diarrhoeic wild boar by culturing the ileum content on VRBG agar plates, characterising the cultured $E$. coli colonies molecularly and RAPD typing them, followed by de novo genome sequencing of a representative $E$. coli isolate designated FKI-5325. RAPD was performed on 37 E. coli isolates cultured from the ileum content. Two RAPD types (RAPD A and B) were identified, which showed $84 \%$ similarity by the Dice coefficient. Isolates assigned to the same RAPD type displayed identical banding patterns. Out of the 37 isolates, 34 (91.8\%) clustered to RAPD type A, corresponding to the dominant $E$. coli O112ab:H2 strain FKI-5325.

The dominant $E$. coli $\mathrm{O} 112 \mathrm{ab}: \mathrm{H} 2$ strain carried the virulence determinants ast $A$ (encoding the EAST1 heatstable toxin), lpfA (long polar fimbrial protein LpfA), gad (glutamate decarboxylase), hlyE (haemolysin E), agn43 (antigen 43 adhesin), and others, while it was negative for the est $A$, estB, stx1, stx2, stx $2 e$, and eae virulence genes. The strain showed intermediate resistance to gentamicin, amikacin, and tobramycin according to the current CLSI disc diffusion zone diameter and MIC breakpoints (6), where the MIC established for gentamicin was $8 \mu \mathrm{g} / \mathrm{mL}$. The strain retained susceptibility to ampicillin, ciprofloxacin, nalidixic acid, tetracycline, and chloramphenicol.
Acquired aminoglycoside resistance genes were not detected in the E. coli $\mathrm{O} 112 \mathrm{ab}: \mathrm{H} 2$ strain and neither were known $16 \mathrm{~S}$ ribosomal RNA gene mutations conferring aminoglycoside resistance.

The observed increased tolerance of $E$. coli FKI5325 to the tested aminoglycosides may be possibly associated with the efflux of aminoglycosides by the AcrD transporter, encoded by the $a c r D$ gene present in its genome $(1,21)$. AcrD provides low-level resistance in $E$. coli to the aminoglycosides amikacin, gentamicin, tobramycin, kanamycin, and neomycin, however, AcrD does not impact minimal inhibitory concentrations (MICs) of other antibiotics including novobiocin, rifampicin, chloramphenicol, carbenicillin, cefoxitin, nalidixic acid, norfloxacin, ampicillin, ciprofloxacin, erythromycin, and tetracycline. It was also shown that a minor increase in the MICs is typical for AcrDmediated resistance to aminoglycosides in E. coli, where MICs of aminoglycosides increased about two- to fourfold over control levels $(1,8,21)$. These findings are in accordance with the level of intermediate aminoglycoside resistance observed for the E. coli FKI5325 strain.

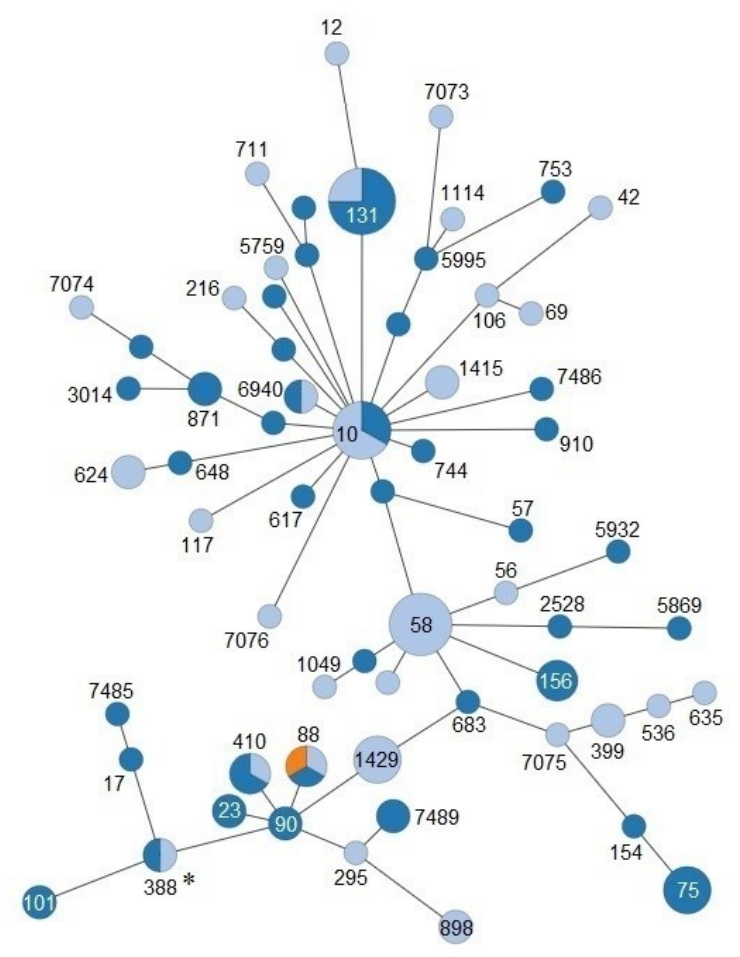

Fig. 2. GrapeTree diagram generated in EnteroBase based on sevengene MLST profiles of $104 \mathrm{E}$. coli strains isolated in Europe from wild boars and domestic pigs $(2,28,29)$. Countries of origin (and number of strains): Denmark (48), Hungary (8), Czech Republic (7), Spain (7), Ireland (6), Luxembourg (5), Italy (4), Switzerland (3), United Kingdom (3), Croatia (2), France (2), Germany (2), Portugal (2), Austria (1), Belgium (1), Bulgaria (1), Netherlands (1), and Sweden (1). Dark blue, light blue, and orange indicate E. coli isolates of domestic pig, wild boar, and food origin, respectively. Numbers stand for sequence types by the Achtman seven-gene MLST scheme (28). The node size in the GrapeTree diagram is proportional to the number of bacterial strains with the same sequence type (ST), and the length of edges are proportional to inter-node genetic distances (29). ST388 determined for the E. coli $\mathrm{O} 112 \mathrm{ab}: \mathrm{H} 2$ strain characterised in this work is marked by an asterisk $(*)$ 
Table 1. Examples of acquired resistance determinants against various antibiotic classes in wastewater metagenomes in Hungary

\begin{tabular}{|c|c|c|c|c|c|c|}
\hline Location & Tetracyclines & Macrolides & Sulphonamides & $\beta$-lactams & Aminoglycosides & NCBI SRA Codes \\
\hline North Pest & tet $W$, tet $O$, tet $M$ & $\begin{array}{l}\text { mefA, } m p h E, \text { ermB, } \\
\text { ermF, erm } G\end{array}$ & sull & bla $_{O X A}$ & $\operatorname{aadA11}, \operatorname{aadA1}, \operatorname{aph}(6)-I d$ & ERR2607463 \\
\hline North Pest & $\begin{array}{l}\text { tet } Q, \text { tet } W, \text { tet } O \text {, } \\
\text { tet } M\end{array}$ & $\begin{array}{l}\text { mefA, mphE, ermB, } \\
\text { ermF, erm } G\end{array}$ & sul1, sul2 & $c f x A$, bla $_{O X A}$, bla $_{T E M}$ & $\operatorname{aadA11}, \operatorname{aph}(6)-I d$ & ERR1713361 \\
\hline
\end{tabular}

Genetic relationships among $E$. coli isolates from wild boars and domestic pigs in Europe. In silico MLST typing in EnteroBase demonstrated that isolate FKI-5325 displayed an allelic profile corresponding to sequence type ST388 (28) and the isolate was assigned to $E$. coli phylogroup B1 (5). The genetic relationships of 104 European E. coli isolates from wild boars and domestic pigs were subjected to minimum spanning tree analysis by the MSTreeV2 tool in EnteroBase and visualised by GrapeTree based on their seven-gene MLST profiles (Fig. 2). E. coli strains sharing the same sequence types were isolated both from domestic pigs and wild boars in Europe (including sequence types ST388, ST10, ST88, ST131, ST410, and ST6940), indicating their potential for dissemination between different habitats and animal populations and contributing thereby to the spread of acquired antibiotic resistance.

Acquired tetracycline, macrolide, and $\beta$-lactam resistance determinants in the gut microbiota of freeliving wild boars in Hungary and Japan. Bioinformatic analysis of shotgun metagenomic contigs of the colon and rectal microbiome of the diarrhoeic wild boar from Hungary identified tet $Q$, tet $W$, and tet $O$ tetracycline resistance genes and the mefA macrolide resistance determinant. These four genes were also detected by PCR and Sanger sequencing in total faecal DNA of four additional free-living wild boars from the Zemplén Mountains, Hungary. Sequence analysis of the metagenomic contigs covering the immediate genetic environment of the tetracycline resistance determinants identified various genes and functions characteristic of mobile genetic elements. These genes included those of an IS1380 family transposase and a TraG conjugal transfer protein for tet $Q$; a relaxase and a mobilisation protein MobC for tet $W$; and a transposon-encoded $\mathrm{TnpV}$ protein and a conjugal transfer protein for tetO (Fig. 3). The wild boar metagenomic contig harbouring tet $Q$ shared a high level of identity $(87 \%-94 \%)$ with two sections of the CTnHyb conjugative transposon of the Bacteroides fragilis strain HMW 615, which also carries a tet $Q$ gene (GenBank accession number KJ816753). The wild boar metagenomic contig harbouring tet $O$ also shared a high level of identity (99\%) with a section of the integrative and conjugative element ICESsuZJ20091101-1 of the Streptococcus suis strain ZJ20091101, which likewise carries a tet $O$ gene (GenBank accession number KX077882).
Functional analysis of the metagenomic contigs obtained for the free-living wild boar from Japan identified a tet $Q$ tetracycline and a $c f x A$-family class-A broad-spectrum $\beta$-lactamase gene in its faecal microbiome.

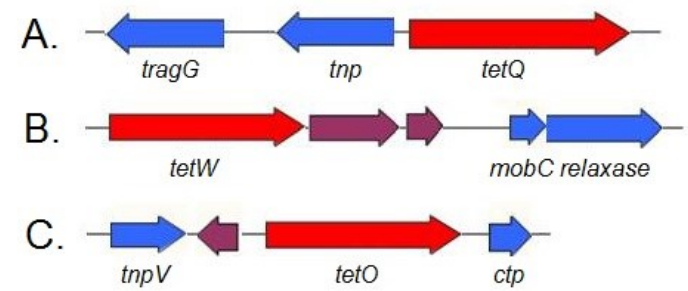

Fig. 3. Immediate genetic environment of the tetracycline resistance determinants identified from the gut microbiome of free-living wild boars in Hungary. Schematic diagrams of metagenomic contigs for the tet $Q(\mathrm{~A})$, tet $W(\mathrm{~B})$, and tet $O$ genes (C), respectively. Abbreviations for selected genes: traG - conjugal transfer protein TraG; tnp transposase; $m o b C$ - mobilisation protein MobC; $\operatorname{tnp} V$ - transposonencoded protein $\mathrm{TnpV}$; and $c t p$ - conjugal transfer protein

Acquired antibiotic resistance determinants in wastewater metagenomes in Hungary. To assess whether the acquired tetracycline and macrolide resistance determinants identified in the gut microbiome of free-living wild boars are also present in environmental samples in Hungary, two wastewater metagenomes of the North-Pest wastewater treatment plant were also analysed by the above bioinformatic methods. These tests indicated the presence of diverse resistance mechanisms including the tet $Q$, tet $W$, tet $O$, and $m e f A$ genes in these environmental metagenomes in Hungary (Table 1), together with various other resistance mechanisms, such as those against aminoglycosides, and in so doing yielded results in accordance with previous findings (15).

\section{Discussion}

The dominance of wild boar and red deer in hunted game can be attributed to their increasing populations in Europe, thus controlling their population numbers requires sustained hunting pressure. Wild boars may be predators of small animals (such as birds, reptiles, small mammals, and arthropods) and can also cause significant damage to vegetation in both natural and agricultural areas where wild boars actively compete for natural resources $(3,7,27)$. 
Land application of sewage sludge or manure from livestock farms containing antibiotic-resistant microbes and antibiotic residues may facilitate the selection and dissemination of resistant strains into both agricultural areas and natural habitats (12). According to surveillance data on the sales of veterinary antimicrobial agents $(4,9)$, the predominant products sold and used both in Europe and Japan for food-producing animals are tetracyclines (Fig. 4). In Hungary and Japan, tetracyclines respectively constituted $46.2 \%$ and $44.1 \%$ of the total sales of active ingredients of veterinary antimicrobial agents for food-producing animals. Significant associations were previously found between country-specific antimicrobial usage and acquired antimicrobial resistance levels in livestock populations (17). The prevalence of the tet $Q$ gene in deer faeces was found to be spatially associated with concentrated animal feeding operations and with land-applied biosolids and livestock manure (20). In a recent study in Europe involving 181 domestic pig farms in nine European countries, the amount of tetracyclines and macrolides used was established to be particularly associated with the abundance of tetracycline and macrolide resistance genes (25), providing robust evidence for the direct selection of resistance genes by these two antibiotic classes.

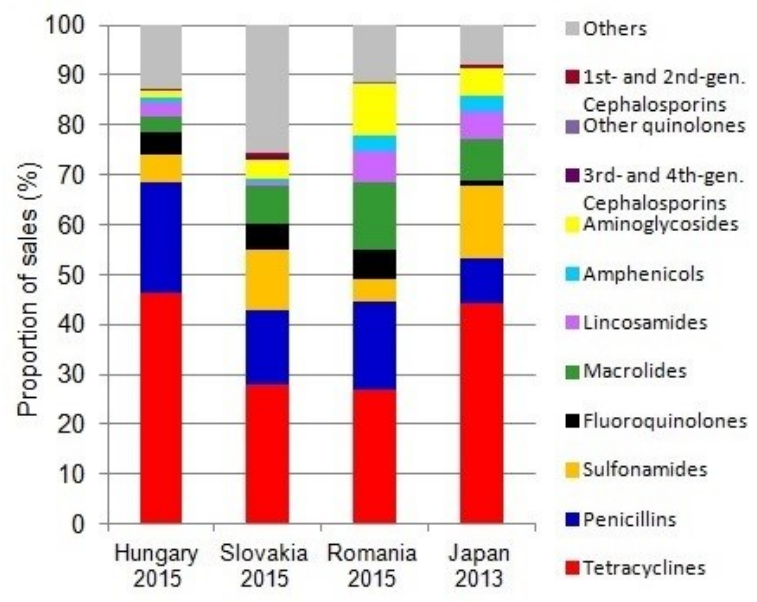

Fig. 4. Proportions of the sales of various veterinary antimicrobial agents in three countries of the European Union in 2015 (9) compared to those in Japan in 2013 for food producing animals (4)

In the present study, tetracycline resistance determinants were identified in the gut microbiome of all examined free-living wild boars. Interestingly, the three tetracycline resistance genes identified in freeliving wild boars from Hungary, tet $Q$, tet $W$, and tet $O$, were overall respectively the first, second, and fourth most abundant tetracycline resistance genes within their antimicrobial class at European domestic pig farms (25). Similarly, the mefA gene detected in our study was overall the most abundant macrolide resistance gene within its antimicrobial class on the 181 European pig farms analysed. Macrolides are used to varying but substantial extents in veterinary medicine in Slovakia, Romania, and Hungary, the three EU countries neighbouring the region of the Zemplén Mountains (Fig. 4). These resistance genes were also detected in wastewater metagenomes from Hungary (Table 1), indicating their presence also in environmental samples.

Concerning the $c f x A$ and tet $Q$ genes detected in the faecal microbiome of the free-living wild boar from Japan, it is notable that although relevant data on domestic animals from Japan were not available to us, a comparative metagenomic analysis of 232 shotgun metagenomes of 10 different natural environment types from five continents indicated that homologs of $c f x A$ were the dominant $\beta$-lactamases in human and bovine faeces samples, indicating anthropogenically impacted environments. The $c f x A$ gene is ubiquitous to bacteroidete isolates from anaerobic human-associated environments (10).

The dominant E. coli O112ab:H2 strain identified in the ileum of the diarrhoeic wild boar harboured the virulence determinant $a s t A$, and displayed sequence type ST388. E. coli clones carrying astA were able to dominate an intestinal section in wild boars, but other E. coli clones, by contrast, were not able to (22). Furthermore, serotype $\mathrm{O} 112$ isolates of E. coli with a similar astA-positive virulence gene profile have also been reported from diarrhoeic domestic pigs from Slovakia and Canada $(18,26)$. The E. coli O112ab:H2 strain belonged to the B1 phylogroup, and it was nonsusceptible to gentamicin, tobramycin, and amikacin. Our results are in accordance with earlier studies suggesting that antibiotic-resistant $E$. coli isolates seem to be more associated with the $\mathrm{B} 1$ and A phylogroups in wild mammals (3). Out of 50 gentamicin-resistant $E$. coli strains of animal or human origin in Hungary, seven animal $E$. coli isolates carried no commonly occurring acquired gentamicin resistance genes (23), similarly to the E. coli FKI-5325 strain characterised in this study.

The observation of diverse acquired antibiotic resistance mechanisms in free-living wild boar populations underscores the need for examination of the impacts of antibiotic use on livestock farms and its effects on the surrounding wildlife populations. Most of the wild animals in this region of Europe share habitat resources with livestock species, and some of them, such as hunted wild boars, are also intended for human consumption. Our data and observations suggest that the prevalence of certain antibiotic resistance genes in the gut microbiome of domestic animals may correlate with their occurrence in free-living wild animal populations. Further, larger-scale studies are needed for assessing epidemiological relationships between antibioticresistant microbiological agents from domestic and wild herds and those from humans, as such research should investigate possible routes of inter-species transfer and routes of transmission at the interfaces of households or farms and the surrounding natural habitats. The increasing overabundance of the European wild boar population amplifies the importance of monitoring its health both in the veterinary and epidemiological aspects and for its role as a zoonotic reservoir. 
Conflict of Interest Statement: The authors declare that there is no conflict of interests regarding the publication of this article.

Financial Disclosure Statement: This study was financially supported by the National Agricultural Research and Innovation Centre (NARIC), Gödöllö, Hungary.

\section{Animal Rights Statement: None required.}

Acknowledgements: The Gróf István Károlyi Hunting Party (Füzérkomlós, Hungary) is acknowledged for providing permission to sample the hunted wild boars in the Zemplén Mountains, Hungary. We would like to thank Gilles Vergnaud, Christine Pourcel, and Tímea Wilk for helpful discussions and suggestions, and Viktor Stéger and Bendegúz Mihalik for providing additional sexual molecular marker data for the wild boars sampled in the Zemplén Mountains in Hungary.

\section{References}

1. Aires J.R., Nikaido H.: Aminoglycosides are captured from both periplasm and cytoplasm by the AcrD multidrug efflux transporter of Escherichia coli. J Bacteriol 2005, 187, 1923-1929.

2. Alikhan N.F., Zhou Z., Sergeant M.J., Achtman M.A.: genomic overview of the population structure of Salmonella. PLoS Genet 2018, 14, e1007261.

3. Alonso C.A., González-Barrio D., Ruiz-Fons F., Ruiz-Ripa L., Torres C.: High frequency of B2 phylogroup among non-clonally related fecal Escherichia coli isolates from wild boars, including the lineage ST131. FEMS Microbiol Ecol 2017, 93, fix016.

4. AMR One Health Surveillance Committee: Nippon AMR One Health Report (NAOR) 2017. Tokyo: Tuberculosis and Infectious Diseases Control Division, Health Service Bureau, Ministry of Health, Labour and Welfare, 2017.

5. Clermont O., Gordon D., Denamur E.: Guide to the various phylogenetic classification schemes for Escherichia coli and the correspondence among schemes. Microbiology 2015, 161, 980-988

6. Clinical and Laboratory Standards Institute (CLSI). Performance Standards for Antimicrobial Susceptibility Testing. $28^{\text {th }}$ ed. CLSI supplement M100. CLSI, 950 West Valley Road, Suite 2500, Wayne, Pennsylvania 19087 USA, 2018.

7. Csányi S., Lehoczi R., Sonkoly K.: National game management database of Hungary. Int J Inform Syst Soc Change 2010, 1, 34-43.

8. Elkins C.A., Nikaido H.: Substrate specificity of the RND-type multidrug efflux pumps AcrB and AcrD of Escherichia coli is determined predominately by two large periplasmic loops. J Bacteriol 2002, 184, 6490-6498.

9. European Medicines Agency, European Surveillance of Veterinary Antimicrobial Consumption, 'Sales of veterinary antimicrobial agents in 30 European countries in 2015'. (EMA/184855/2017), 2017

10. Gatica J., Jurkevitch E., Cytryn E.: Comparative metagenomics and network analyses provide novel insights into the scope and distribution of B-lactamase homologs in the environment. Front Microbiol 2019, 10, 146.

11. Heijnen L., Medema G.: Quantitative detection of E. coli, E. coli O157 and other shiga toxin producing $E$. coli in water samples using a culture method combined with real-time PCR. J Water Health 2006, 4, 487-498.
12. Heuer H., Schmitt H., Smalla K.: Antibiotic resistance gene spread due to manure application on agricultural fields. Curr Opin Microbiol 2011, 14, 236-243.

13. Holman D.B., Brunelle B.W., Trachsel J., Allen H.K.: Metaanalysis to define a core microbiota in the swine gut. mSystems 2017, 2, e00004-17.

14. Klose V., Bayer K., Kern C., Goelß F., Fibi S., Wegl G.: Antibiotic resistances of intestinal lactobacilli isolated from wild boars. Vet Microbiol 2014, 168, 240-244.

15. Libisch B., Uzinger N., Biró B., Anton A.: Isolation and characterisation of potentially pathogenic antibiotic-resistant bacterial strains from sewage sludge samples in Hungary. In: Book of Abstracts: The $11^{\text {th }}$ European Meeting on Environmental Chemistry, 8-11 December 2010, Portorož, Slovenia, edited by P. Trebše, M. Petrič, V. Lavtižar, University of Nova Gorica, Nova Gorica, 2010, p. 193.

16. Literak I., Dolejska M., Radimersky T., Klimes J., Friedman M., Aarestrup F.M., Hasman H., Cizek A.: Antimicrobial-resistant faecal Escherichia coli in wild mammals in central Europe: multiresistant Escherichia coli producing extended-spectrum $\beta$-lactamases in wild boars. J Appl Microbiol 2010, 108, 1702-1711.

17. Munk P., Knudsen B.E., Lukjancenko O., Duarte A.S.R., Luiken R.E.C., Van Gompel L., Smit L.A.M., Schmitt H., Garcia A.D., Hansen R.B., Petersen T.N., Bossers A., Ruppe E., Lund O., Hald T., Pamp S.J., Vigre H., Heederik D., Wagenaar J.A., Mevius D., Aarestrup F.M.: Abundance and diversity of the faecal resistome in slaughter pigs and broilers in nine European countries. Nat Microbiol 2018, 3, 898-908.

18. Ngeleka M., Pritchard J., Appleyard G., Middleton D.M., Fairbrother J.M.: Isolation and association of Escherichia coli AIDA-I/STb, rather than EAST1 pathotype, with diarrhea in piglets and antibiotic sensitivity of isolates. $J$ Vet Diagn Invest 2003, 15, 242-252.

19. Pacheco A.B.F., Guth B.E.C., De Almeida D.F., Ferreira L.C.S.: Characterization of enterotoxigenic Escherichia coli by random amplification of polymorphic DNA. Res Microbiol 1996, 147, 175-182.

20. Rogers S.W., Shaffer C.E., Langen T.A., Jahne M., Welsh R.: Antibiotic-resistant genes and pathogens shed by wild deer correlate with land application of residuals. Ecohealth 2018, 15, 409-425.

21. Rosenberg E.Y., Ma D., Nikaido H.: AcrD of Escherichia coli is an aminoglycoside efflux pump. J Bacteriol 2000, 182, 1754-1756.

22. Schierack P., Römer A., Jores J., Kaspar H., Guenther S., Filter M., Eichberg J., Wieler L.H.: Isolation and characterization of intestinal Escherichia coli clones from wild boars in Germany. Appl Environ Microbiol 2009, 75, 695-702.

23. Szmolka A., Anjum M.F., La Ragione R.M., Kaszanyitzky É.J., Nagy B.: Microarray based comparative genotyping of gentamicin resistant Escherichia coli strains from food animals and humans. Vet Microbiol 2012, 156, 110-118.

24. Ushida K., Tsuchida S., Ogura Y., Toyoda A., Maruyama F.: Domestication and cereal feeding developed domestic pig-type intestinal microbiota in animals of suidae. Anim Sci J 2016, 87, 835-841.

25. Van Gompel L., Luiken R.E., Sarrazin S., Munk P., Knudsen B.E., Hansen R.B., Mevius D.J., Bossers A., Aarestrup F.M., Dewulf J., Wagenaar J.A., Mevius D.J., Schmitt H., Heederik D.J.J., DoradoGarcia A., Smit L.A.M.: The antimicrobial resistome in relation to antimicrobial use and biosecurity in pig farming, a metagenome-wide association study in nine European countries. J Antimicrob Chemother 2019, 74, 865-876.

26. Vu-Khac H., Holoda E., Pilipcinec E., Blanco M., Blanco J.E., Dahbi G., Mora A., Lopez C., Gonzalez E.A., Blanco J.: Serotypes, virulence genes, intimin types and PFGE profiles of Escherichia coli isolated from piglets with diarrhoea in Slovakia. Vet J 2007, 174, 176-187.

27. Wasyl D., Zając M., Lalak A., Skarżyńska M., Samcik I., Kwit R., Jablonski A., Bocian L., Wozniakowski G., Hoszowski A., 
Szulowski K.: Antimicrobial resistance in Escherichia coli isolated from wild animals in Poland. Microb Drug Resist 2018, 24, 807-815.

28. Wirth T., Falush D., Lan R., Colles F., Mensa P., Wieler L.H., Karch H., Reeves P.R., Maiden M.C., Ochman H., Achtman M.: Sex and virulence in Escherichia coli: an evolutionary perspective. Mol Microbiol 2006, 60, 1136-1151.

29. Zhou Z., Alikhan N.F., Sergeant M.J., Luhmann N., Vaz C., Francisco A.P., Carrico J.A., Achtman M.: GrapeTree: visualization of core genomic relationships among 100,000 bacterial pathogens. Genome Res 2018, 28, 1395-1404.

30. Zottola T., Montagnaro S., Magnapera C., Sasso S., De Martino L., Bragagnolo A., D'Amici L., Condoleo R., Pisanelli G., Iovane G., Pagnini U.: Prevalence and antimicrobial susceptibility of Salmonella in European wild boar (Sus scrofa); Latium Region - Italy. Comp Immunol Microbiol Infect Dis 2013 , $36,161-168$. 